\title{
Nitric Oxide Selectively Tunes Inhibitory Synapses to Modulate Vertebrate Locomotion
}

\author{
David L. McLean and Keith T. Sillar \\ School of Biology, Division of Biomedical Sciences, University of St. Andrews, St. Andrews, FIFE KY16 9TS, \\ United Kingdom
}

We have explored the possible modulation by nitric oxide (NO) of inhibitory synaptic transmission mediated by either glycine or GABA during episodes of rhythmic fictive swimming in postembryonic Xenopus laevis tadpoles. Extracellular ventral-root recordings suggest a stage-dependent increase in the reliability and extent of the NO donor $S$-nitroso- $n$-acetylpenicillamine (SNAP; 0.1-1 mM) to inhibit swimming by reducing the frequency and shortening the duration of swim episodes. These effects of SNAP on the swimming rhythm at both developmental stages are corroborated by intracellular recordings from presumed motor neurons with sharp microelectrodes, which also suggest that NO inhibits swimming by facilitating both glycinergic and GABAergic inhibition. However, we found no

The output of neural networks that control locomotor behaviors depends on the electrical properties of the constituent neurons and the relative weightings of their synaptic interconnections. There is now a substantial body of evidence demonstrating that these properties in motor networks can be adapted through neuromodulation (Katz, 1999). Neuromodulation often involves the activation of metabotropic receptors that couple to second messenger pathways, whose targets bias motor networks toward a particular output configuration. The selectivity in the effects of a given modulator will obviously depend on the features of its release and its receptors. More recent evidence has begun to reveal another form of neuromodulation, termed volume transmission (Vizi, 1984; Iversen and Goodman, 1986; Dawson and Snyder, 1994), in which it less easy to understand how specificity can be achieved (but see Davisson et al., 1997; Ohta et al., 1997). One notable example is the gas nitric oxide (NO) which is generated after the breakdown of L-arginine to L-citrulline (Palmer et al., 1988) by the enzyme nitric oxide synthase (NOS). NO is highly diffusible and reaches its intracellular targets rapidly, without the requirement for membrane receptors on the surface of candidate neurons. The main target for NO is the soluble form of guanylyl cyclase (Arnold et al., 1977), but it is clear that NO can also influence ion-channel function (Ohkuma et al., 1998) and thereby directly change the electrical properties of neurons.

\footnotetext{
Received Nov. 9, 2001; revised Jan. 28, 2002; accepted Feb. 21, 2002.

This work was supported by the Biotechnology and Biological Sciences Research Council and The Wellcome Trust. We thank Dr. Anthony R. Butler (School of Chemistry, University of St. Andrews) for providing the nitric oxide donor SNAP.

Correspondence should be addressed to Dr. Keith T. Sillar, School of Biology, Division of Biomedical Sciences, Bute Medical Buildings, University of St. Andrews, St. Andrews, FIFE KY16 9TS, UK. E-mail: kts1@st-andrews.ac.uk.

Copyright (C) 2002 Society for Neuroscience $0270-6474 / 02 / 224175-10 \$ 15.00 / 0$
}

evidence for NO modulation of the excitatory drive for swimming. In addition to presynaptic effects on inhibitory transmitter release, a pronounced postsynaptic membrane depolarization ( $\sim 5-10 \mathrm{mV})$ and conductance decrease ( $10-20 \%)$ are associated with bath application of SNAP. Hence, NO exerts inhibitory effects on swimming through multiple but selective actions on both the electrical properties of spinal neurons and on particular synaptic interconnections. The presynaptic and postsynaptic effects of $\mathrm{NO}$ act in concert to tune inhibitory synapses.

Key words: nitric oxide; GABA; glycine; spinal cord; release; locomotion

We have recently explored the distribution of nitrergic neurons and the role of $\mathrm{NO}$ in the control of locomotion in a simple vertebrate, tadpoles of the clawed frog Xenopus laevis (stage 42) (Nieuwkoop and Faber, 1956). We have shown in this model that NOS is present in putative locomotive centers in the brainstem, where it affects locomotion early in Xenopus development (McLean and Sillar, 2000, 2001). NO appears to play an inhibitory role in the modulation of the swimming circuitry, slowing swim frequency and shortening the duration of swim episodes (McLean and Sillar, 2000). However, the ways in which the inhibitory actions of NO are accomplished have yet to be described.

The two known fast inhibitory neurotransmitters that influence spinal swimming circuitry in Xenopus tadpoles originate from the glycinergic commissural interneurons (Dale et al., 1986) and the GABAergic midhindbrain reticulospinal (mhr) neurons (Roberts et al., 1987). The glycinergic interneurons generate the fast midcycle IPSPs that couple the two sides of the spinal cord in alternation during swimming. The strength of midcycle inhibition is a major determinant of cycle period (Dale, 1995; Soffe, 1987), in which increased midcycle inhibition results in increased cycle periods and vice versa. In contrast, the GABAergic mhr neurons mediate a stopping reflex in hatchling Xenopus embryos (stage 37/38). When the rostral cement gland contacts an obstacle, swimming ceases abruptly through the release of GABA from mhr neurons onto spinal motor networks (Boothby and Roberts, 1992a,b). The reliability of this response decreases during larval development (Boothby and Roberts, 1992a), but it is believed that the mhr neurons become incorporated into an endogenous stopping mechanism in larvae (Reith and Sillar, 1999). The slowing of swim frequency and shortening of swim episodes elicited by NO are thus consistent with a facilitation of glycinergic and GABAergic inhibition, respectively. The purpose of this study was to 
$a_{1}$

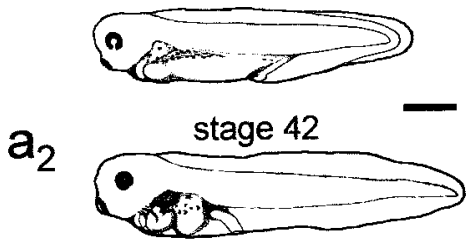

b

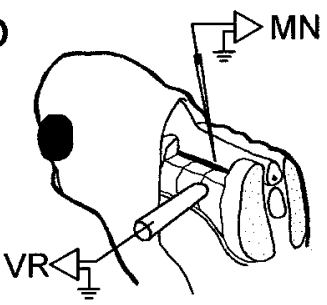

$c_{1}$
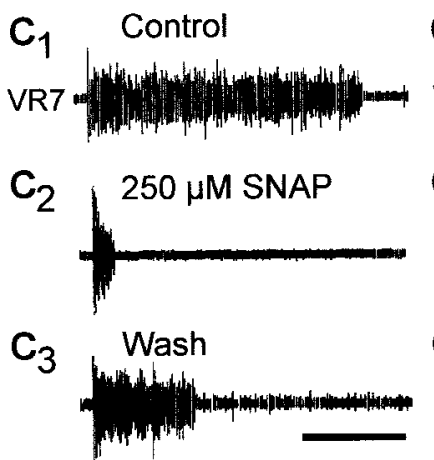

$d_{3}$
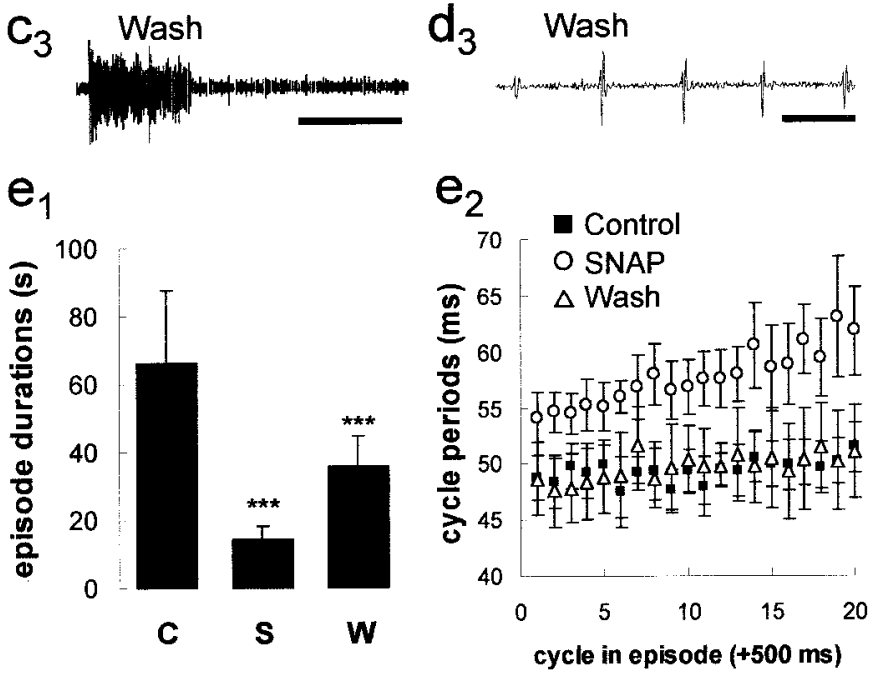

Figure 1. The X. laevis tadpole preparation and the effects of NO on fictive swimming. $a$, Postembryonic Xenopus tadpoles at the two stages $\left(a_{1}, 37 / 38 ; a_{2}, 42,\right)$ used in this study. Scale bar, $1 \mathrm{~mm}$. $b$, A schematic drawing illustrating an extracellular ventral root $(V R)$ recording from an intermyotomal cleft (only one illustrated) and an intracellular recording from a presumed motor neuron $(M N)$. For more details, see Materials and Methods. Bath application of SNAP can reversibly shorten swimming episode durations $\left(c_{1}-c_{3}\right)$ and slow swimming frequency $\left(d_{1}-d_{3}\right)$. Calibration: $c, 15 \mathrm{sec} ; d, 50 \mathrm{msec} . e_{1}$, Bar graph representing pooled data $( \pm$ SEM) illustrating that SNAP reversibly shortens the duration of episodes $(n=12)$. $C$, Control; $S$, SNAP; $W$, wash. ***p $<0.05 . e_{2}$, Scatter graph of 20 cycles of swimming activity representing the pooled data ( \pm SEM) from 12 experiments illustrating that SNAP reversibly lengthened cycle periods $(n=12)$. Drawings in $a_{1}$ and $a_{2}$ were adapted from Nieuwkoop and Faber (1956).

investigate the inhibitory mechanisms responsible for the effects of NO on the Xenopus swimming system.

\section{MATERIALS AND METHODS}

Experimental preparations. All experiments were performed on late embryonic (stage 37/38) and early larval (stage 42) X. laevis tadpoles (Fig. $1 a_{1}, a_{2}$ ) obtained by induced breeding from an adult laboratory colony and staged according to Nieuwkoop and Faber (1956). Animals were first anesthetized in $0.1-1 \%$ tricaine methanesulfonate, immobilized in 12.5 $\mu \mathrm{M} \alpha$-bungarotoxin (Sigma, Poole, UK), and then secured in a chamber with recirculating frog Ringer solution [ionic composition in mM: 115 $\mathrm{NaCl}, 2.5 \mathrm{KCl}, 1 \mathrm{MgCl}_{2}, 2.4 \mathrm{NaHCO}_{3}, 10 \mathrm{HEPES}, 2 \mathrm{CaCl}_{2}$ (for extracellular experiments) or $4 \mathrm{CaCl}_{2}$ (for intracellular experiments), $\mathrm{pH} 7.4$; $20-22^{\circ} \mathrm{C}$ ]. Fictive motor patterns appropriate to drive swimming behav- ior were elicited by brief $1 \mathrm{msec}$ current pulses (DS2-type isolated stimulator; Digitimer, Welwyn Garden City, UK) to the flank skin and were recorded from ventral roots in the intermyotomal clefts with glass suction electrodes after removal of the flank skin from the otic capsule to the anus. For intracellular recordings, the overlying myotomes were removed and recordings were made from neurons positioned in the ventral quarter of the spinal cord (Fig. $1 b$ ), where motor neurons predominate (Roberts and Clarke, 1982). Penetrations were made with microelectrodes pulled from $1 \mathrm{~mm}$ borosilicate glass capillary tubing (CEI Harvard Apparatus, Reading, UK) with direct current resistances of $\sim 90-150 \mathrm{M} \Omega$ when filled with $3 \mathrm{M} \mathrm{KCl}$. KCl-filled electrodes were selected to make chloride-dependent IPSPs strongly depolarizing (Fig. $\left.2 a_{1}-a_{3}\right)$ and hence easier to measure. In addition, IPSPs mediated by glycine and $\mathrm{GABA}_{\mathrm{A}}$ receptors can be readily distinguished on the basis of their durations (glycine, $\sim 50 \mathrm{msec}$; $\mathrm{GABA}_{\mathrm{A}}, \sim 100-200 \mathrm{msec}$ ) as well as their pharmacological sensitivities (Reith and Sillar, 1997).

Drugs. Drugs were bath applied by adding known quantities to the stock bottle to achieve the desired final bath concentration. $S$-nitroso-nacetylpenicillamine (SNAP) and $n$-acetylpenicillamine (NAP) were made fresh daily and dissolved in $0.01 \%$ dimethylsulfoxide (DMSO). Strychnine, bicuculline, and tetrodotoxin (TTX) were dissolved in distilled water and subsequently frozen at $-20^{\circ} \mathrm{C}$ in stock solutions until required. All drugs were purchased from Sigma, with the exception of SNAP, which was supplied by the School of Chemistry at the University of St. Andrews.

To control for any nonspecific vehicle-mediated effects (cf. Hedrick and Morales, 1999), experiments with the vehicle DMSO (0.1-1\%) and the inactive isomer of SNAP, NAP (0.1-1.25 mM) were performed. Bath application of NAP had no significant effects $(n=4 ; p>0.05$; paired $t$ test) on any of the measured experimental parameters (Table 1). In addition, DMSO $(n=4 ; p>0.05$; paired $t$ test $)$ had no pronounced effects on the synaptic drive for swimming as recorded from motor neurons (Table 1), consistent with the lack of vehicle effects on the parameters of swimming in larvae (McLean and Sillar, 2000).

Data analysis. Data were recorded and displayed conventionally and stored on videotape using a pulse code modulator adapter (Medical Systems Corp., Greenvale, NY). Hard-copy records were made off-line using a thermal chart recorder (Graphtec, Tokyo, Japan) or digital plotter (Gould Instruments, Hainault, UK). Data analysis was performed off-line using Dataview (courtesy of William J. Heitler, University of St. Andrews, St. Andrews, UK) and the Spike 2 analysis software package (Cambridge Electronic Design, Cambridge, UK). For each experiment, three consecutive episodes of swimming activity were measured in control, drug, and wash conditions. For measurements within swimming episodes, the first $500 \mathrm{msec}$ of activity in each episode was ignored, to avoid possible influences arising directly from sensory stimulation. In addition, before measuring the synaptic drive during swimming in the presence of SNAP, sufficient levels of tonic hyperpolarizing current were first injected into the cell to compensate for the SNAP-induced membrane potential depolarization. Two statistical tests were used to determine significant differences between conditions. The parametric paired $t$ test was used in instances in which data were compared between experiments. The nonparametric Mann-Whitney $U$ test was used when sample sizes differed. Significance was determined at $p<0.05$ and unless stated otherwise, data are given as means \pm SEM.

\section{RESULTS}

\section{Stage-independent modulation of fictive swimming activity by nitric oxide}

The effects of NO on stage 42 larval swimming activity have been documented previously (McLean and Sillar, 2000). In addition, the developmental expression of NO synthase in brainstem neuron populations suggests that the role of NO in swimming may be relatively constant during larval life (McLean and Sillar, 2001). However, there are profound developmental differences between swimming activity in late embryos and early larvae (Sillar et al., 1992a,b). Therefore, in this study we first investigated whether NO had the same effects on swimming at the earlier, hatching stage 37/38 (Fig. 1 $a_{1}$ ), during which intracellular recordings are easier and the synaptic drive for swimming is less complex (Sillar et al., 1992a). The durations of swimming episodes recorded from embryonic preparations in control conditions were on average 

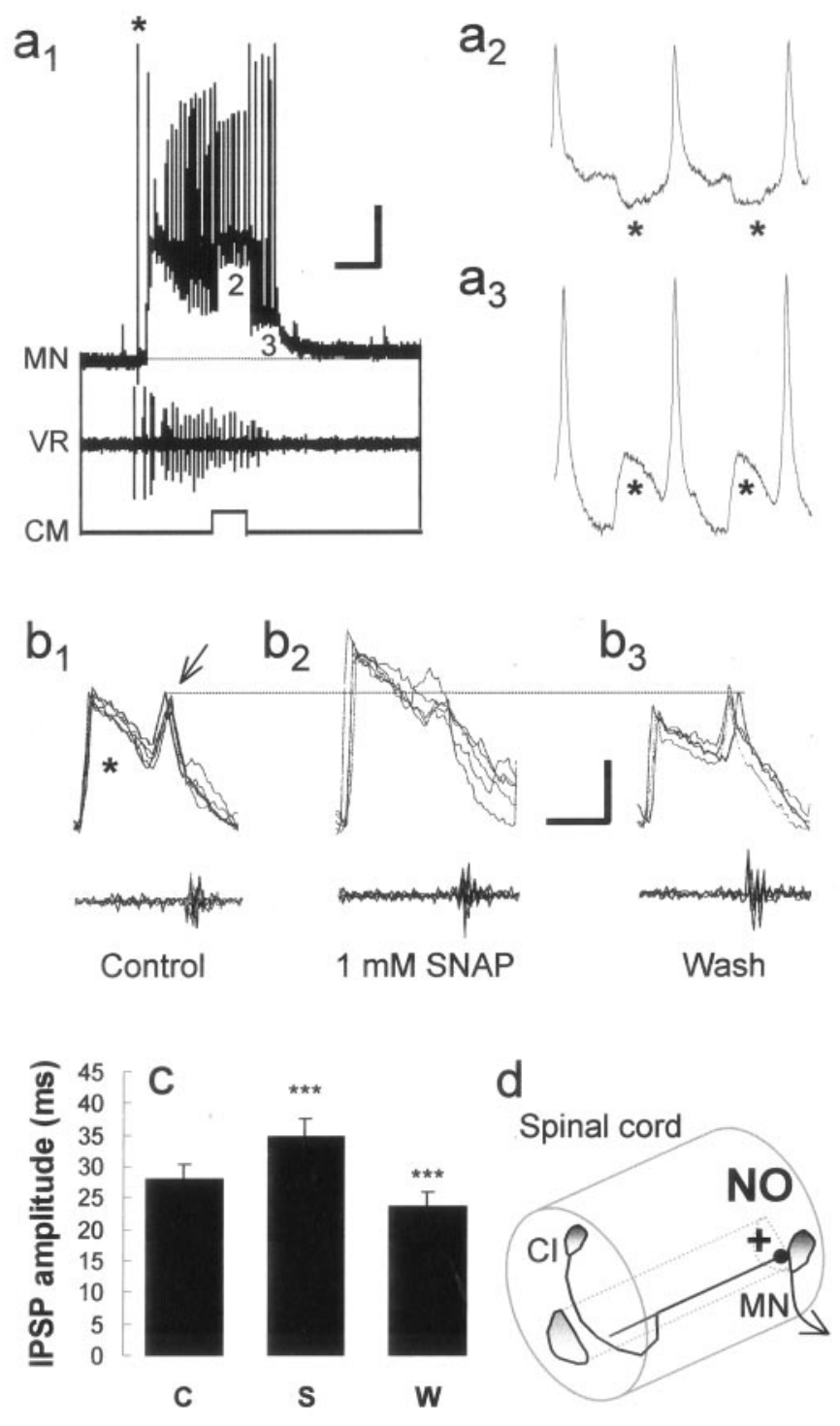

Figure 2. NO potentiates midcycle inhibition. $a_{1}$, The synaptic drive to motor neurons $(M N \mathrm{~s})$ during swimming consists of a gradually declining tonic depolarization, on which on-cycle excitation [coincident with ventral root $(V R)$ activity] and midcycle inhibition (interposed between VR activity) are superimposed. KCl-filled microelectrodes render midcycle IPSPs depolarizing ( $a_{3}$; see Materials and Methods), but a sufficient amount of positive current injection causes the inhibition to become hyperpolarizing again $\left(a_{2}\right)$. The dotted line in $a_{1}$ represents resting potential before stimulation. $C M$, Current monitor. Asterisks indicate stimulation artifacts. Calibration: $10 \mathrm{mV}$ for voltage in $a_{1}-a_{3}$ and $0.1 \mathrm{nA}$ for current in $a_{1} ; 500 \mathrm{msec}$ in $a_{1}$ and $20 \mathrm{msec}$ in $a_{2}-a_{3} . b$, Bath application of $1 \mathrm{mM}$ SNAP results in a pronounced increase in midcycle IPSP amplitudes $\left(b_{1}-b_{2}\right)$, which is fully reversible after returning to control saline $\left(b_{3}\right)$. This is illustrated by trains of five consecutive superimposed cycles of intracellular $(M N)$ and extracellular $(V R 7)$ activity, illustrated on a faster time scale. Note the midcycle IPSP (asterisk) and the on-cycle EPSP (arrow). Note also the increased inhibitory shunting of the excitatory component of the synaptic drive under SNAP (dotted line). Calibration: $10 \mathrm{mV}, 20 \mathrm{msec} . c$, The bar graph represents the pooled data ( \pm SEM) from 18 experiments, which illustrate that SNAP reversibly increased midcycle IPSP amplitudes. $d$, A schematic drawing illustrates a commissural interneuron $(C I)$ and the possible site of the nitrergic potentiation of glycine release onto motor neurons in the spinal cord. $C$, Control; $S$, SNAP; $W$, wash. ${ }^{* * *} p<0.05$.

\begin{tabular}{|c|c|c|c|c|c|c|}
\hline \multirow[b]{2}{*}{ Effects/drugs } & \multicolumn{3}{|c|}{ Stage $37 / 38$} & \multicolumn{3}{|c|}{ Stage 42} \\
\hline & Inc & Dec & $\mathrm{N} / \mathrm{S}$ & Inc & Dec & $\mathrm{N} / \mathrm{S}$ \\
\hline \multicolumn{7}{|l|}{ Membrane properties } \\
\hline \multicolumn{7}{|l|}{ Conductance } \\
\hline SNAP & 0 & 4 & 1 & 0 & 4 & 1 \\
\hline TTX/SNAP & 2 & 0 & 0 & 2 & 0 & 0 \\
\hline NAP & 0 & 0 & 1 & 0 & 0 & 3 \\
\hline DMSO & 0 & 0 & 4 & 0 & 0 & 0 \\
\hline \multicolumn{7}{|l|}{ Resting potential } \\
\hline SNAP & 10 & 0 & 1 & 6 & 0 & 1 \\
\hline TTX/SNAP & 2 & 0 & 0 & 2 & 0 & 0 \\
\hline NAP & 0 & 0 & 1 & 0 & 0 & 3 \\
\hline DMSO & 0 & 0 & 4 & 0 & 0 & 0 \\
\hline \multicolumn{7}{|l|}{ IPSP occurrence } \\
\hline \multicolumn{7}{|l|}{ Quiescent } \\
\hline SNAP & 9 & 0 & 2 & 6 & 0 & 1 \\
\hline Bicuc/SNAP & 1 & 0 & 0 & 2 & 0 & 0 \\
\hline Strych/SNAP & 3 & 0 & 0 & 3 & 0 & 0 \\
\hline Strych/Bicuc/SNAP & 0 & 0 & 2 & 0 & 0 & 0 \\
\hline TTX/SNAP & 2 & 0 & 0 & 2 & 0 & 0 \\
\hline NAP & 0 & 0 & 1 & 0 & 0 & 3 \\
\hline DMSO & 0 & 0 & 4 & 0 & 0 & 0 \\
\hline \multicolumn{7}{|l|}{ Synaptic drive } \\
\hline \multicolumn{7}{|l|}{ Midcycle IPSPs } \\
\hline SNAP & 6 & 1 & 4 & 6 & 0 & 1 \\
\hline Strych/SNAP & 0 & 0 & 3 & 0 & 0 & 3 \\
\hline NAP & 0 & 0 & 1 & 0 & 0 & 3 \\
\hline DMSO & 0 & 0 & 4 & 0 & 0 & 0 \\
\hline \multicolumn{7}{|l|}{ Terminating IPSPs } \\
\hline SNAP & 6 & 0 & 5 & 5 & 0 & 2 \\
\hline Strych/SNAP & 1 & 0 & 2 & 3 & 0 & 0 \\
\hline NAP & 0 & 0 & 1 & 0 & 0 & 3 \\
\hline DMSO & 0 & 0 & 4 & 0 & 0 & 0 \\
\hline
\end{tabular}

${ }^{a}$ Significant increase (Inc), decrease (Dec), or no significant change (N/S) $(p<0.05$ considered significant). Bicuc, Bicuculline; Strych, strychnine.

$66.4 \pm 21.4 \mathrm{sec}$, but were significantly $(p<0.05$; paired $t$ test $)$ reduced to $14.1 \pm 4.1 \mathrm{sec}$ in the presence of the NO donor SNAP $(n=12 ; 0.1-1 \mathrm{mM})$. Episode durations increased significantly $(p<0.05$; paired $t$ test $)$ to $35.7 \pm 8.9 \mathrm{sec}$ by washing the preparations in fresh, control saline (Fig. $1 c_{1}-c_{3}, e_{1}$ ). These pooled data were also analyzed separately to determine how consistently SNAP decreased episode durations in individual experiments, for comparison with previous extracellular experiments at stage 42 (McLean and Sillar, 2000). As a result, it was clear that in $67 \%$ of experiments $(n=8)$ SNAP could significantly shorten episode durations, whereas in the remaining experiments $(n=4)$ there was no significant effect. Therefore, NO could shorten the duration of swim episodes in embryos as it does in larvae, albeit not as reliably as reported previously for larval preparations $(\sim 90 \%)$ (McLean and Sillar, 2000). Similarly, NO could significantly $(p<$ 0.05 ; paired $t$ test) increase cycle periods during swimming in embryonic preparations, from on average $49.4 \pm 2.3 \mathrm{msec}$ to $55.8 \pm 2.8$ msec cycle periods (Fig. $1 d_{1}-d_{3}, e_{2}$ ). This effect could be significantly ( $p<0.05$; paired $t$ test) reversed to $48.3 \pm 2.4$ after washing in fresh, control saline. Again, this was similar to, but not as reliable as, the effect on cycle periods documented in larvae $(\sim 80 \%$ ) (McLean and Sillar, 2000), because SNAP significantly 
increased the cycle period in only $50 \%$ of embryonic experiments $(n=6)$, whereas in the remainder $(n=6)$ it did not. There was no correlation between the presence or absence of a clear effect on embryonic swimming and the concentration of SNAP that was applied in this study, which was similar to the concentration range used at larval stages (100-500 $\mu \mathrm{M})$ (McLean and Sillar, 2000). This suggests that the concentrations applied were saturating for the preparations that responded, at both developmental stages. Thus, NO appears to perform a similar function at these two stages of development, albeit more consistently at the larval stage. Therefore, we explored the effects of NO on the membrane potential changes of motor neurons during swimming at these two stages and found the results to be comparable at each stage (Table 1).

\section{Effects of nitric oxide on synaptic drive underlying swimming}

The synaptic drive to motor neurons during episodes of swimming in hatchling $X$. laevis tadpoles consists of a gradually declining tonic depolarization (Fig. $2 a_{1}$ ), on which fast on-cycle excitation, triggering a single spike per cycle, and fast midcycle (glycinergic) inhibition are superimposed (Fig. $2 a_{2}-a_{3}$ ) (cf. Kahn and Roberts, 1982). The midcycle inhibition is normally hyperpolarizing (Fig. $2 a_{2}$ ) but can be made strongly depolarizing when KCl-filled electrodes are used (Fig. 2a3). This drives a simple motor pattern at stage $37 / 38$, which matures in a rostrocaudal sequence by stage 42 (Fig. $1 a_{2}$ ), coincident with the invasion of the spinal networks by descending serotonergic axons (van Mier et al., 1986; Sillar et al., 1995). The result is that within $24 \mathrm{hr}$ after hatching, motor neurons are able to fire several action potentials per cycle in a much more variable motor pattern (Sillar et al., 1992a).

\section{Nitric oxide modulates inhibitory components of the synaptic drive for swimming}

Bath application of SNAP ( $n=18 ; 0.1-1 \mathrm{~mm})$ significantly $(p<$ 0.05 ; paired $t$ test) increased the amplitudes of glycinergic midcycle IPSPs from $28.1 \pm 2.2$ to $34.7 \pm 2.9 \mathrm{mV}$ (Fig. $2 b_{1}-b_{3}, c$ ), as determined from the pooled data of both embryos and larvae (Table 1). A wash in fresh, control saline returned midcycle IPSP amplitudes to $\sim 23.5 \pm 2.4 \mathrm{mV}$. This effect on midcycle IPSP amplitudes was also correlated with a significant increase in cycle periods from, on average, $53.4 \pm 1.8 \mathrm{msec}$ to $58.0 \pm 1.8 \mathrm{msec}(n=$ $18 ; p<0.05$; paired $t$ test). Midcycle IPSPs are mediated by the inhibitory amino acid transmitter glycine, released from the synaptic terminals of commissural interneurons (Dale et al., 1986; Soffe, 1987) (Fig. 2d), and are a major determinant of cycle period (Dale, 1995). Accordingly, midcycle amplitudes were abolished by the application of strychnine $(n=6 ; p<0.05$; paired $t$ test $)$, leaving only the tonic depolarization at midcycle (Fig. $3 b$, dotted line), and the subsequent application of SNAP had no effect at midcycle (Fig. $3 d$ ), nor did it slow swimming (compare Fig. $\left.3 a_{1}-a_{3}\right)$. For example, in experiments in which strychnine eliminated midcycle IPSPs $(n=6)$, there was also a small but significant shortening of cycle periods from $50.1 \pm 1.5 \mathrm{msec}$ to $48.1 \pm$ $1.5 \mathrm{msec}$ ( $p<0.05$; paired $t$ test). After the subsequent addition of SNAP, cycle periods were on average $48.5 \pm 2.5 \mathrm{msec}$ and did not differ significantly ( $p>0.05$; paired $t$ test). These clear effects on the amplitudes of midcycle glycinergic IPSPs during swimming were not paralleled by equivalent changes in the excitatory drive for swimming. For instance, in the presence of strychnine to eliminate any effects on the midcycle IPSPs $(n=6)$, the remain-

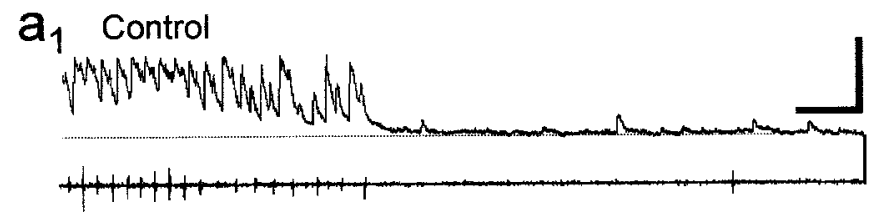

\section{$a_{2} 2 \mu \mathrm{M}$ Strychnine}

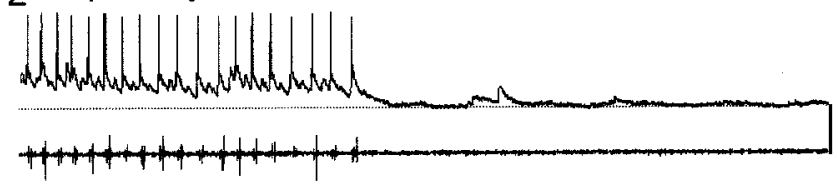

$a_{3}+500 \mu$ M SNAP
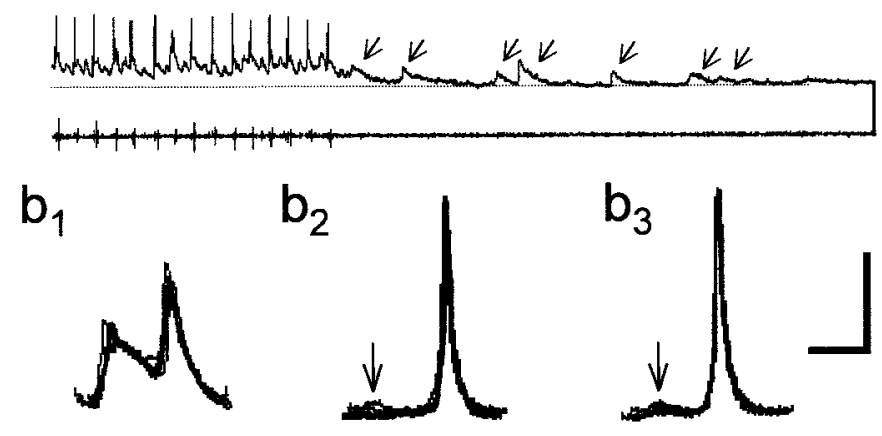

Control

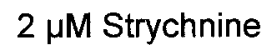

+1 mM SNAP
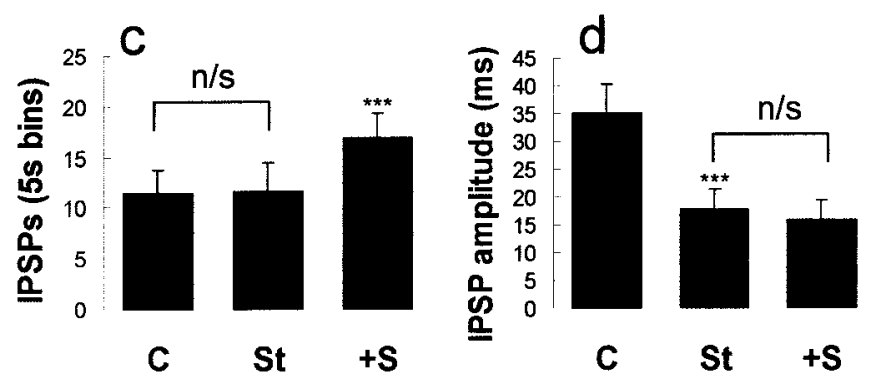

Figure 3. NO selectively potentiates strychnine-sensitive and strychnineinsensitive components of swimming. $a$, The facilitation of presumed GABAergic IPSPs by SNAP was confirmed by a test of their pharmacological sensitivity with the glycine antagonist strychnine. In control conditions, there is still a clear midcycle component to swimming $\left(a_{1}\right)$ that is eliminated by bath application of $2 \mu \mathrm{M}$ strychnine $\left(a_{2}\right)$. Note that diminution of the midcycle inhibition "releases" spiking during on-cycle excitation. Subsequent application of $500 \mu \mathrm{M}$ SNAP potentiates longerduration GABAergic IPSPs that can terminate swimming episodes (arrows; $a_{3}$ ). Calibration: $50 \mathrm{mV}, 300 \mathrm{msec} . b$, The complete elimination of midcycle IPSPs by strychnine (arrows) is also illustrated in five overlapping traces of consecutive cycles on a faster time scale $\left(b_{1}-b_{2}\right)$. However, there is still a measurable tonic depolarization, denoted by the dotted line. The subsequent application of SNAP has no obvious effect at midcycle or on-cycle synaptic inputs $\left(b_{3}\right)$. Note that this also illustrates the shunting effect of inhibition on excitation (compare Fig. $2 b$ ). Calibration: $25 \mathrm{mV}, 25 \mathrm{msec} . c$, Bar graph of the pooled data from six experiments ( \pm SEM) illustrating that SNAP increased the occurrence of GABAergic IPSPs at the end of swim episodes in the presence of strychnine. $d$, This graph illustrates the pooled data $( \pm$ SEM) from six experiments in which SNAP was unable to increase the amplitude of midcycle IPSP in the presence of strychnine. Note that strychnine does not completely eliminate the amplitude because a proportion represents the tonic NMDAmediated depolarization. ***p $<0.05$. $C$, Control; St, strychnine; $+S$, plus SNAP; $n / s$, not significantly different $(p>0.05)$. 

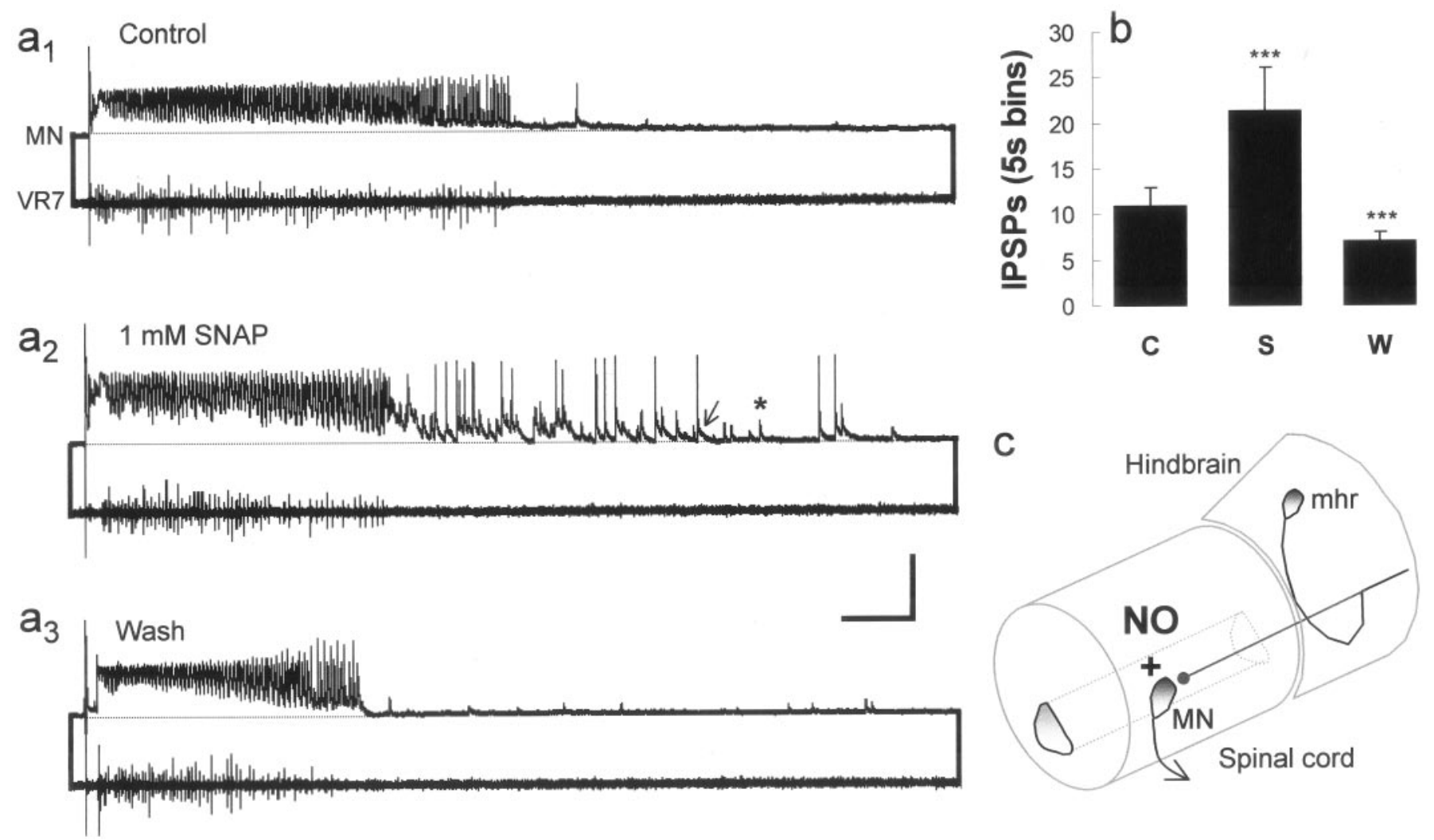

Figure 4. NO potentiates the inhibition that terminates swimming. $a$, Simultaneous intracellular [motor neuron $(M N)]$ and extracellular [ventral root $7(V R 7)]$ recordings of fictive swimming illustrate that episodes can terminate spontaneously $\left(a_{1}\right)$, yet the subsequent application of 1 mM SNAP reversibly increases the occurrence of GABAergic IPSPs that can terminate swimming $\left(a_{2}-a_{3}\right)$, which are distinguished based on duration (glycine, asterisk; GABA, arrow). Calibration: $50 \mathrm{mV}, 1 \mathrm{sec}$. Note that traces are illustrated and measurements were made at similar resting membrane potentials (see Materials and Methods for additional details). The dotted line indicates resting membrane potential before stimulation. $b$, The bar graph illustrates the pooled data $( \pm$ SEM) from 18 experiments in which SNAP reversibly increased GABAergic IPSPs and is expressed as the mean number of IPSPs measured $5 \mathrm{sec}$ after the termination of an episode of swimming. $c$, A schematic drawing illustrates a GABAergic mhr neuron and the possible site of action for nitrergic potentiation of GABA release onto motor neurons in the spinal cord.

ing tonic NMDA receptor-mediated depolarization was not significantly altered by SNAP (Fig. 3d). The fast on-cycle excitation was suprathreshold in the presence of strychnine and SNAP, obscuring any possible small effects of SNAP on the underlying EPSP. However, on-cycle impulses (Fig. $3 b_{1}-b_{3}$ ) measured in the presence of strychnine were on average $53.4 \pm 0.9 \mathrm{msec}$ and after application of SNAP were $51.8 \pm 1.4 \mathrm{msec}(n=6 ; p>0.05$; paired $t$ test); thus, the excitatory drive during swimming was not detectably affected.

\section{Nitric oxide potentiates GABAergic termination of swimming}

Embryonic swim episodes normally terminate spontaneously (Fig. $4 a_{1}$ ), whereas larval swimming episodes often, but not always, end with a barrage of GABAergic IPSPs (Reith and Sillar, 1999) reminiscent of those elicited by cement gland stimulation in embryos (Boothby and Roberts, 1992a,b) (Fig. 4c). GABAergic IPSPs are easily distinguished from glycinergic ones on the basis of their duration (Fig. 5d,e) (Reith and Sillar, 1997). These IPSPs are also known to be GABAergic because they disappear in the presence of the $\mathrm{GABA}_{\mathrm{A}}$ receptor antagonist bicuculline (Reith and Sillar, 1999), yet they persist in the presence of the glycine antagonist strychnine (Fig. $6 a_{5}$ ). After bath application of SNAP, there was a significant increase $(n=18 ; p<0.05$; paired $t$ test) in the frequency of GABAergic IPSPs from $10.9 \pm 2.1$ to $21.4 \pm 3.4$ recorded within $5 \mathrm{sec}$ of the end of a swimming episode (Fig. $\left.4 a_{1}-a_{3}, b\right)$. This returned to $7.0 \pm 1.3$ IPSPs per $5 \mathrm{sec}$ bin (or time window) after a wash in fresh, control saline. In larvae, the frequency of GABAergic IPSPs that often, but not always, occurred at the end of episodes was increased in the presence of SNAP (data not shown), whereas in embryos, GABAergic IPSPs occurred when previously there were none (Fig. 4a). This increase in IPSP occurrence was also correlated with a decrease in average episode durations, from $12.4 \pm 3.4$ to $4.5 \pm 0.9 \mathrm{sec}(n=$ 18 ; $p<0.05$; paired $t$ test). In addition, bath application of SNAP potentiated the occurrence of GABAergic IPSPs within $5 \mathrm{sec}$ of the end of episodes in the presence of strychnine, from $11.6 \pm 4.6$ to $16.9 \pm 6.5$ (Fig. $3 a_{1}-a_{3}, c$ ). Furthermore in these experiments, SNAP decreased the average duration of swimming episodes in the presence of strychnine from $3.3 \pm 0.6 \mathrm{sec}$ to $2.7 \pm 0.5 \mathrm{sec}(n=$ 6; $p<0.05$; paired $t$ test).

\section{Nitric oxide increases spontaneous IPSP rate}

In the quiescent periods between swimming episodes, frequent depolarizing IPSPs are recorded using KCl-filled electrodes (Fig. 5a) (Reith and Sillar, 1997). In addition to the effect of NO on the inhibitory synaptic drive during swimming, there was also a pronounced increase in the frequency of these spontaneous IPSPs. Within the first 5 min of bath application, SNAP caused a pronounced and reversible (Fig. $5 a, b, c, f$ ) increase in the occurrence of depolarizing IPSPs from $5.3 \pm 1.3$ IPSPs per $2 \mathrm{sec}$ bin to $7.9 \pm 1.5$ IPSPs per $2 \sec$ bin $(n=18 ; p<0.05$; paired $t$ test $)$. 
Figure 5. NO produces an increase in spontaneous IPSP occurrence. $a$, During interepisode quiescent periods, depolarizing IPSPs can be distinguished that have been shown previously to be TTXresistant, reflecting spontaneous GABA and glycine release from presynaptic terminals (Reith and Sillar, 1997). b, Shortly (2-3 min) after bath application of $500 \mu \mathrm{M}$ SNAP, there was a profound and reversible (c) increase in the occurrence of GABAergic and glycinergic IPSPs, which could be distinguished on the basis of their duration (compare $d$ and $e$ ). Calibration: $a-c, 40 \mathrm{mV}$, $500 \mathrm{msec} ; d, e, 20 \mathrm{mV}, 100 \mathrm{msec}$. Note the impulse (arrow) triggered off the glycinergic IPSP. $f$, SNAP produces a significant $(* * * p<0.05)$ and reversible increase in the occurrence of IPSPs. A bar graph of the pooled data $( \pm$ SEM) from the 18 experiments illustrates that SNAP reversibly increased IPSP occurrence and is constructed from $30 \mathrm{sec}$ of continuous data measured in 2 sec bins under each condition. $C$, Control; $S$, SNAP; $W$, wash.
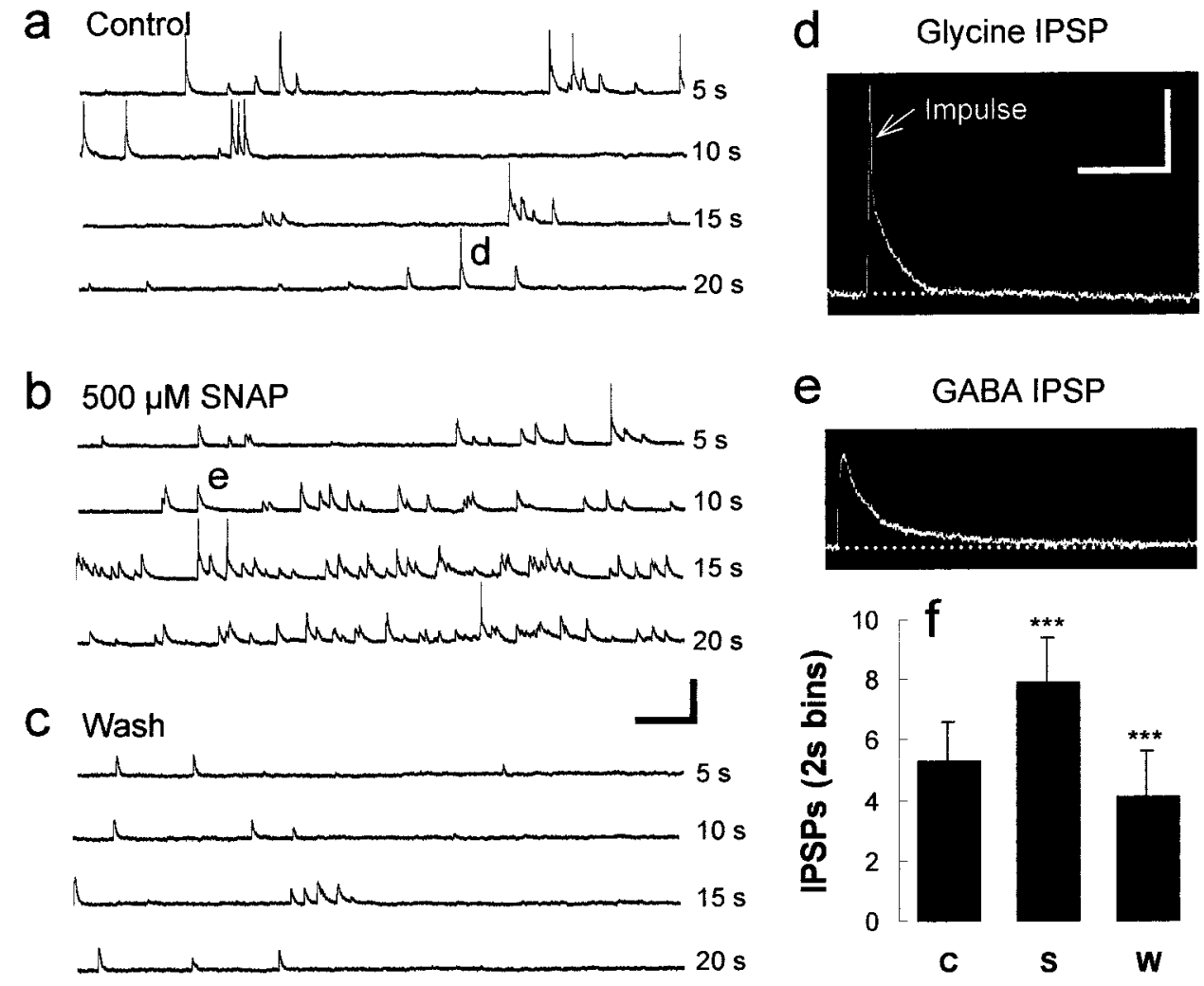

These values returned to $4.0 \pm 1.5$ after a wash in fresh, control saline. The spontaneous inhibitory potentials comprise a mixture of both glycinergic (Fig. 5d) and GABAergic (Fig. 5e) IPSPs that are easily distinguishable based on their duration (Reith and Sillar, 1997). SNAP appeared to increase the rate of occurrence of both types, but to confirm this, the pharmacology of these IPSPs was also investigated using strychnine $(1-2 \mu \mathrm{M} ; n=6)$ and bicuculline $(20-50 \mu \mathrm{M} ; n=3)$. In control conditions, both glycinergic and GABAergic IPSPs were present (Fig. $6 a_{1}$ ). Bath application of $50 \mu \mathrm{M}$ bicuculline selectively eliminated the GABAergic IPSPs (Fig. $6 a_{2}$ ), whereas the subsequent bath application of 500 $\mu \mathrm{M}$ SNAP increased the frequency of the remaining shorterduration, presumably glycinergic IPSPs (Fig. $6 a_{3}, b_{1}$ ). After removal of SNAP (wash) (Fig. $6 a_{4}$ ) and after the application of $2 \mu \mathrm{M}$ strychnine to the same preparation, only longer-duration GABAergic IPSPs could be detected (Fig. $6 a_{5}$ ); the subsequent application of SNAP elicited an additional increase in spontaneous activity (Fig. $6 a_{6}, b_{2}$ ). Furthermore, in the presence of both strychnine and bicuculline ( $n=2 ; p>0.05$; paired $t$ test), SNAP did not affect any remaining spontaneous, very infrequent depolarizing, presumably excitatory PSPs (Fig. 6c). In summary, these experiments suggest a facilitation by $\mathrm{NO}$ of inhibitory synaptic pathways that could be accounted for by a direct presynaptic enhancement of the probability of transmitter release.

\section{Effects of nitric oxide on inhibitory synapses are presynaptic}

To investigate whether NO can directly modulate the release machinery in inhibitory synapses, the effects of NO on TTXresistant quantal glycinergic and GABAergic IPSPs were examined. Under TTX, as in the quiescent periods between swimming episodes, spontaneous depolarizing synaptic potentials fall into distinct size categories, presumably reflecting the quantal release of glycine (Wall and Dale, 1993; McDearmid et al., 1997) and
GABA (Reith and Sillar, 1997, 1999). The presence of TTXresistant IPSPs allowed a direct investigation of presynaptic versus postsynaptic sites of nitrergic modulation, in which effects on IPSP frequency indicate presynaptic mechanisms and effects on IPSP amplitudes indicate a postsynaptic site of action. In the presence of TTX $(n=4)$, bath application of SNAP produced an increase in IPSP frequency $(\sim 1.75 \pm 0.35$ per $2 \mathrm{sec}$ bin $)$. However, bath application of SNAP often produced a concomitant membrane potential depolarization (Fig. $7 a_{1}$ ). Therefore, although the amplitude distribution of IPSPs at depolarized levels attained under SNAP did not vary in the presence of SNAP (data not shown), there was a clear increase in IPSP amplitudes $(p<$ 0.05; Mann-Whitney $U$ test) (Fig. 7a ) when the membrane potential was returned to control values by negative current injection, an effect that was also apparent in the absence of TTX (Fig. 7b). Thus, although these data confirm that NO can act presynaptically to modulate the release machinery of inhibitory synapses, they also clearly indicate parallel postsynaptic effects.

\section{Parallel effects of nitric oxide on motor neuron membrane properties}

Finally, we examined the effects of SNAP on the membrane properties of presumed motor neurons. A pronounced $5-10 \mathrm{mV}$ ( $n=10$; mean, $6.7 \pm 1.5 \mathrm{mV} ; p<0.05$; paired $t$ test) membrane potential depolarization was observed shortly after bath application of SNAP both under control conditions (Fig. 7b) and as described above with TTX (Fig. $7 a_{1}$ ). To determine whether the depolarization was attributable to changes in the ionic conductance of the motor neuron, hyperpolarizing conductance pulses were applied in 10 experiments before, during, and after SNAP application. There was a significant $10-20 \%$ conductance decrease ( $n=10$; mean, $16.0 \pm 4.0 \% ; p<0.05$; paired $t$ test) with SNAP compared with controls (Fig. $7 c_{1}, c_{3}$ ), an effect that was reversed after returning to control saline (Fig. $7 d_{1}$ ). A similar 

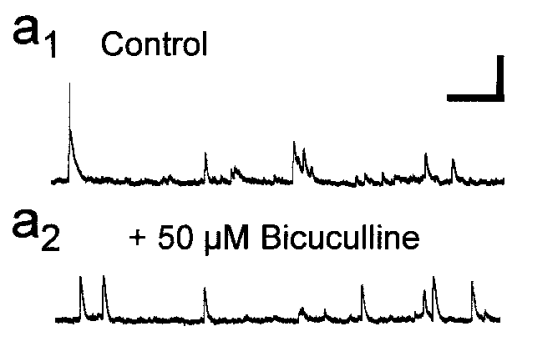

$a_{3}$
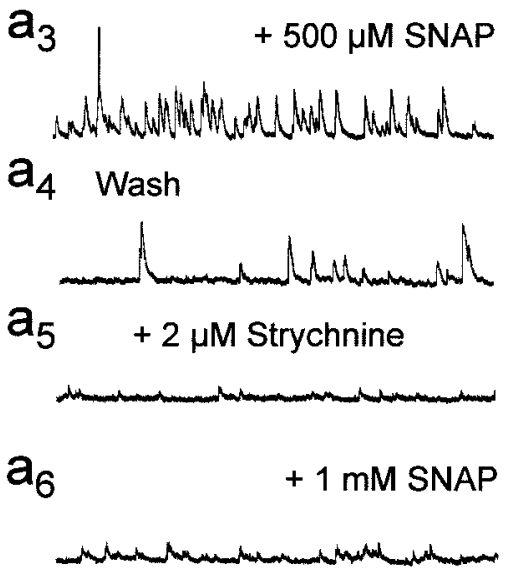

GABAergic synapses in the spinal cord and direct effects on the membrane potential and conductance of spinal motor neurons. The increase in spontaneous glycinergic and GABAergic IPSPs between episodes is paralleled by the facilitation of the inhibitory synaptic drive during and at the termination of swimming in both embryos and larvae. Specifically, bath-applied SNAP can reversibly increase the amplitude of glycinergic midcycle IPSPs during swimming and the incidence of GABAergic IPSPs at the end of episodes. The reliability of these effects increases from stage $37 / 38$ to stage 42 (Table 1 ). In combination, they can account for the slowing and shortening of swimming episodes in the presence of NO, confirming interpretations of previous extracellular findings (McLean and Sillar, 2000). However, at present it cannot be determined at how many other levels NO is acting, whether directly on GABAergic and glycinergic synapses in the spinal cord, indirectly on the firing of inhibitory neurons, or possibly in the brainstem via modulation of the activity of aminergic neurons, which in turn affect inhibitory synapses. The presence of NOS in brainstem neuronal populations (McLean and Sillar, 2001) and not in the spinal cord at these stages of development suggests that the latter is plausible.

\section{Effects of nitric oxide on membrane potential and conductance}

Bath application of SNAP led to a pronounced and reliable membrane potential depolarization associated with a conductance decrease. Simultaneous membrane depolarization and conductance decrease is consistent with the closure of a positively charged outward current, which implicates a $\mathrm{K}^{+}$conductance. There are several outward $\mathrm{K}^{+}$currents present in Xenopus tadpole spinal neurons (Dale and Kuenzi, 1997). However, the relative contributions of these currents change with development (Sun and Dale, 1998), and the membrane depolarization and conductance decrease described here are persistent at both developmental stages (Table 1). Furthermore, any reduction of these currents contributes to multiple spiking and spike broadening, which leads to excitatory effects on the swimming rhythm (Dale and Kuenzi, 1997). However, there is a small conductance $\mathrm{Ca}^{2+}$-dependent $\mathrm{K}^{+}$current that is not subject to developmental regulation and is thought to contribute to the termination of swimming episodes (Wall and Dale, 1995). Regardless, if a $\mathrm{K}^{+}$ conductance is blocked by NO, the channels bear a striking resemblance to members of the two-pore-domain "leak" $\mathrm{K}^{+}$ channel family. These contribute to the resting membrane potential and thus shape the characteristics of neuronal excitability (Talley et al., 2001). In particular, one family subset, tandem pore domain acid-sensitive $\mathrm{K}^{+}$channel-1, is known to be a target for neuromodulation in motor neurons (Talley et al., 2000). NO interaction with $\mathrm{K}^{+}$channels has been described in other preparations (Ahern et al., 1999), but the exact nature and function of any NO interaction with $\mathrm{K}^{+}$conductances in Xenopus tadpoles awaits further investigation. Of course, any NO-induced closure of $\mathrm{K}^{+}$conductance in presynaptic targets to the motor neurons could potentially lead to the increased excitability of, and firing in, commissural and mhr interneurons and thus to the increased glycine and GABA transmitter release described here. However, for a number of reasons we think this is unlikely to be the only explanation. First, there would presumably be the depolarization and activation of excitatory neurons as well as inhibitory ones, yet NO selectively potentiates inhibition. Second, the potentiation of inhibitory transmitter release persists in the presence of TTX, which would not be possible if NO were working solely through

These experiments using intracellular recordings suggest that NO has several significant effects on the spinal circuitry for swimming, including facilitation of transmission at both glycinergic and 
Figure 7. NO elicits a membrane potential depolarization and a conductance decrease, which persists in the presence of TTX. $a_{1}$, In the presence of $100 \mathrm{~nm}$ TTX, the spontaneous increase in IPSP frequency and amplitude elicited by SNAP is apparent as the membrane begins to depolarize. $a_{2}$, This is further quantified as a bar graph constructed from IPSP amplitudes measured within 30 continuous seconds in this experiment. As such, there were more data points in the SNAP condition, so the Mann-Whitney $U$ test was used to determine significance. $* * * p<0.05$. Calibration: $20 \mathrm{mV}, 30 \mathrm{sec} . b$, A recording from a motor neuron $(M N)$ in the absence of TTX on a slower time base also illustrates the membrane depolarization elicited by bath application of $1 \mathrm{~mm}$ SNAP. Note the episodes of spontaneous swimming activity and the increased occurrence of IPSPs during interepisode periods in the presence of SNAP, which increase in amplitude when the membrane potential is returned to control values after tonic hyperpolarizing current injection. $C M$, Current monitor. Trains of five superimposed $100 \mathrm{msec}$ hyperpolarizing conductance test pulses under control saline with $\left(c_{1}\right)$ and without $\left(c_{2}\right)$ a $0.1 \mathrm{nA}$ negative holding current show no detectable change in membrane conductance, despite a $10 \mathrm{mV}$ difference in membrane potential. After the application of $1 \mathrm{~mm}$ SNAP, the membrane potential depolarizes and a pronounced decrease in membrane conductance is observed $\left(c_{3}, c_{4}\right)$ that appears to be independent of membrane potential (compare values in $c_{1}$ and $c_{3}$ ). Differing levels of membrane potential are aligned for ease of comparison (dotted line). Note that at depolarized levels, a small rebound potential can occur (asterisk). This was also observed at similar membrane potentials in control conditions and therefore is unlikely to reflect a SNAP effect. Calibrations: $b, 10 \mathrm{mV}, 30 \mathrm{sec}, 0.1 \mathrm{nA} ; c, 5 \mathrm{mV}, 80 \mathrm{msec} . d_{1}$, SNAP produced a significant (*** $p<0.05$ ) decrease in membrane conductance, which persisted in the presence of $100 \mathrm{~nm}$ TTX $\left(d_{2}\right)$. The bar graphs of the pooled data $( \pm$ SEM) from 10 experiments in the absence of TTX and 4 experiments in the presence of TTX illustrate that SNAP reversibly decreased conductance and are expressed as percentage differences from control values. $C$, Control; $S$, SNAP; $W$, wash.

the excitation of network interneurons. Finally, if the NOinduced depolarization does occur in presynaptic inhibitory terminals, it is possible that this is sufficient to open voltagedependent calcium channels and increase calcium entry and in this way facilitate transmitter release.

\section{The reliability of nitrergic facilitation of inhibition increases during development}

It is clear from extracellular evidence that NO functions very much like a brake, slowing and then stopping swimming (McLean and Sillar, 2000). Therefore, it is reasonable to assume that NO has a net inhibitory effect on the spinal swim circuitry. This effect, as illustrated here, appears to follow a developmental gradient in that the reliability with which NO modulates swimming behavior increases as the animal matures. This suggests that NO is performing its inhibitory role via pathways that are also developmentally regulated. In support, the brainstem mhr GABA pathway that terminates swimming when the cement gland is activated in embryos changes during larval development, coincident with the degeneration of the cement gland. By stage 42, episodes of swimming often terminate with a barrage of GABA IPSPs without any stimulation of the cement gland (Reith and Sillar, 1999); presumably the mhr neurons are rewired into an intrinsic stopping pathway on which NO can now have an effect. The nature of the midcycle glycinergic inhibition also changes during the same period of development; in embryos the inhibition is a single compound IPSP that varies little in time course or amplitude from one cycle to the next. However, by stage 42, the midcycle inhibition comprises multiple IPSPs whose amplitude varies on a cycle-by-cycle basis (Sillar et al., 1992a). This change also provides the substrate for NO to have a more pronounced effect at the larval than at the embryonic stage.

There is now considerable evidence to suggest that the normal maturation and development of locomotor circuitry depends on functionally intact descending inputs (for review, see Vinay et al., 2000), at least some of which are nitrergic in this species (McLean and Sillar, 2001). Therefore, it is likely that higher centers, whose descending axons invade the spinal cord in a developmentally regulated manner, are somehow involved in the nitrergic modulation described here. In support, the number of nitrergic neurons appears to increase in strict developmental sequence in the brainstem, in discrete populations that are interconnected and that also have descending axons (McLean and Sillar, 2001). Therefore, it would not be unreasonable to assume that NO is working in concert with other, more conventional, neurotransmitters.

\section{Relationship between nitric oxide and brainstem neuromodulators}

The descending serotonergic innervation of the spinal cord from the raphe region is not only causally linked to the maturation of the swimming locomotor pattern in Xenopus (Sillar et al., 1995), but it also appears to be engaged in the continuous modulation of swimming (Sillar et al., 1992b). The serotonergic effects on swimming oppose those of another biogenic amine, noradrenaline (NA), via actions on a common cellular target, namely inhibitory glycinergic commissural interneurons, which control the strength of midcycle inhibition (McDearmid et al., 1997). It is conceivable that NO alters midcycle IPSP amplitudes by modu- 


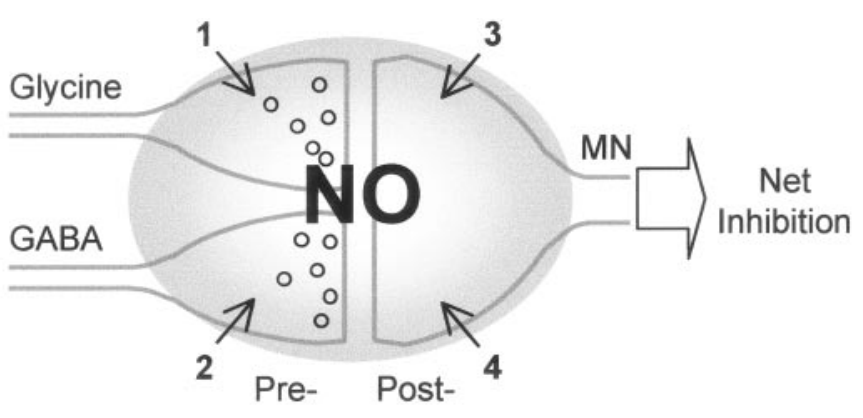

1. Increased reciprocal inhibition = slow swimming

2. Increased descending inhibition = short episodes

3. Membrane potential depolarisation

4. Decreased membrane conductance

Inhibition more potent

Figure 8. The volume transmitter NO yields a net inhibitory effect on locomotion via presynaptic and postsynaptic mechanisms. This schematic summary diagram lists all of the effects $\mathrm{NO}$ has on the spinal circuitry for swimming, primarily by facilitation of glycine and GABA release and the modification of motor neuron $(M N)$ membrane properties. Open circles represent synaptic vesicles.

lating the release of these neuromodulators, particularly because our recent evidence using NADPH-diaphorase staining suggests colocalization of NOS with aminergic neurons in the brainstem (McLean and Sillar, 2000, 2001). In relation to this idea, NO is known to inhibit the synthesis of 5-HT from tryptophan hydroxylase (Kuhn and Arthur, 1997) and can inactivate 5-HT itself (Fossier et al., 1999). In addition, in the amphibian tadpole of the related anuran species, Rana temporaria, NA and NO both initiate qualitatively identical motor patterns (McDearmid and Sillar, 1997); in rat spinal synaptosomes, NO has been implicated in the facilitation of NA release (Li et al., 2000). The combined promotion of NA release and inhibition of 5-HT release by NO would result in an increase in glycine transmission, and hence an increase in cycle periods.

The duration of swim episodes appears to be controlled at least in part by the activity of a known GABAergic stopping pathway, involving the brainstem $\mathrm{mhr}$ neurons (Boothby and Roberts, 1992b). If NO synthase were present in GABAergic mhr neurons as we have speculated (McLean and Sillar, 2000), then activation of these neurons during a normal stopping response could trigger the release of $\mathrm{NO}$ via $\mathrm{Ca}^{2+}$-dependent pathways. NO could then act in a retrograde manner on synaptic inputs from higher centers, reinforcing appropriate connections in a classical "Hebbian" manner (Gally et al., 1990). It would seem logical for NO to act as a trigger for activating the mhr neurons to stop swimming endogenously after periods of intense activity, given that production can be activity-dependent (Dawson and Snyder, 1994; Sasaki et al., 2000). NO could promote GABA release not only retrogradely but also anterogradely by promoting the fusion of synaptic vesicles (Meffert et al., 1994, 1996). Either or both of these nonexclusive proposals would certainly explain the facilitation of GABA release by NO.

Regardless of the interplay between NO and other transmitters in the brainstem, the net effects of NO on swimming involve facilitation of glycinergic and GABAergic inhibition to produce a decrease in swim frequency and a decrease in swim episode durations, respectively. This is corroborated by intracellular recordings from presumed motor neurons under SNAP, showing facilitation of glycinergic and GABAergic components of the synaptic drive for swimming. In addition, the present experiments revealed parallel postsynaptic effects of NO in a prominent membrane potential depolarization and conductance decrease, both of which will complement the presynaptic facilitation of inhibitory transmitter release (Fig. 8). First, the depolarization will take the membrane potential further away from the normal reversal potential for the IPSP (close to rest) so that the IPSPs will become larger. Second, the conductance decrease will have the effect of increasing the responsiveness of the motor neurons to transmitter release so that even IPSPs of equivalent amplitude will have a larger effect under NO. The key to the net inhibitory effect of NO on swimming is that it selectively potentiates inhibitory synapses through parallel presynaptic and postsynaptic mechanisms (Fig. 8). These results provide important new insights to the role of NO in vertebrate motor control.

\section{REFERENCES}

Ahern GP, Hsu SF, Jackson MB (1999) Direct actions of nitric oxide on rat neurohypophysial $\mathrm{K}^{+}$channels. J Physiol (Lond) 520:165-176.

Arnold WPCKM, Katsuki S, Murad F (1977) Nitric oxide activates guanylate cyclase and increases guanosine $3^{\prime}: 5^{\prime}$-cyclic monophosphate levels in various tissue preparations. Proc Natl Acad Sci USA 74:3203-3207.

Boothby KM, Roberts A (1992a) The stopping response of Xenopus laevis embryos: behaviour, development and physiology. J Comp Physiol [A] 170:171-180.

Boothby KM, Roberts A (1992b) The stopping response of Xenopus laevis embryos: pharmacology and intracellular physiology of rhythmic spinal neurons and hindbrain neurons. J Exp Biol 169:65-86.

Dale N (1995) Experimentally derived model for the locomotor pattern generator in the Xenopus embryo. J Physiol (Lond) 489:489-510.

Dale N, Kuenzi FM (1997) Ion channels and the control of swimming in the Xenopus embryo. Prog Neurobiol 53:729-756.

Dale N, Ottersen OP, Roberts A, Storm-Mathisen J (1986) Inhibitory neurons of a motor pattern generator in Xenopus revealed by antibodies to glycine. Nature 324:255-257.

Davisson RL, Travis MD, Bates JN, Johnson AK, Lewis S (1997) Stereoselective actions of $S$-nitrosocysteine in central nervous system of conscious rats. Am J Physiol 272:H2361-H2368.

Dawson TM, Snyder SH (1994) Gases as biological messengers: nitric oxide and carbon monoxide in the brain. J Neurosci 10:5147-5159.

Fossier P, Blanchard B, Ducrocq C, LePrince C, Tauc L, Baux G (1999) Nitric oxide transforms serotonin into an inactive form and this affects neuromodulation. Neuroscience 93:597-603.

Gally JA, Montague PR, Reeke GN, Edelman GM (1990) The NO hypothesis: possible effects of a short-lived, rapidly diff usible signal in the development and function of the nervous system. Proc Natl Acad Sci USA 87:3547-3551.

Hedrick MS, Morales RD (1999) Nitric oxide as a modulator of central respiratory rhythm in the isolated brainstem of the bullfrog (Rana catesbeiana). Comp Biochem Physiol [A] 124:243-251.

Iversen LL, Goodman EC (1986) Fast and slow chemical signaling in the nervous system. New York: Oxford UP.

Kahn JA, Roberts A (1982) The central nervous origin of the swimming motor pattern in embryos of Xenopus laevis. J Exp Biol 99:185-196.

Katz PS (1999) Beyond neurotransmission: neuromodulation and its importance for information processing. Oxford: Oxford UP.

Kuhn DM, Arthur Jr R (1997) Molecular mechanism of the inactivation of tryptophan hydroxylase by nitric oxide: attack on critical sulfhydryls that spare the enzyme iron center. J Neurosci 17:7245-7251.

Li XH, Rose G, Dongre N, Pan HL, Tobin JR, Eisenach JC (2000) $S$-nitroso-L-cysteine releases norepinephrine in rat spinal synaptosomes. Brain Res 872:301-307.

McDearmid JR, Sillar KT (1997) A slow non-rhythmic motor pattern elicited by both noradrenaline and nitric oxide in embryos of the frog Rana temporaria. J Physiol (Lond) 504:12P.

McDearmid JR, Scrymgeour-Wedderburn JFS, Sillar KT (1997) Aminergic modulation of glycine release in a spinal network controlling swimming. J Physiol (Lond) 503:1473-1482.

McLean DL, Sillar KT (2000) The distribution of NADPH-diaphoraselabelled interneurons and the role of nitric oxide in the swimming system of Xenopus laevis larvae. J Exp Biol 203:693-704.

McLean DL, Sillar KT (2001) Spatiotemporal pattern of nicotinamide adenine dinucleotide phosphate-diaphorase reactivity in the developing central nervous system of premetamorphic Xenopus laevis tadpoles. J Comp Neurol 437:350-362.

Meffert MK, Premack BA, Schulman H (1994) Nitric oxide stimulates $\mathrm{Ca}^{2+}$-independent synaptic vesicle release. Neuron 12:1235-1244.

Meffert MK, Calakos NC, Scheller RH, Schulman H (1996) Nitric oxide 
modulates synaptic vesicle docking/fusion reactions. Neuron 16:1229-1236.

Nieuwkoop PD, Faber J (1956) Normal tables for Xenopus laevis (Daudin). Amsterdam: North Holland.

Ohkuma S, Katsura M, Hibino Y, Xu J, Shirotani K, Kuriyama K (1998) Multiple actions of nitric oxide on voltage-dependent $\mathrm{Ca}^{2+}$ channels in mouse cerebral cortical neurons. Brain Res Mol Brain Res 54:133-140.

Ohta H, Bates J, Lewis S, Talman W (1997) Actions of $S$-nitrosocysteine in the nucleus tractus solitarii are unrelated to the release of nitric oxide. Brain Res 746:98-104.

Palmer RMJ, Ashton DS, Moncada S (1988) Vascular endothelial cells synthesize nitric oxide from L-arginine. Nature 333:664-666.

Reith CA, Sillar KT (1997) Pre- and postsynaptic modulation of spinal GABAergic neurotransmission by the neurosteroid, $5 \beta$-pregnan-3 $\alpha$-ol20-one. Brain Res 770:202-212.

Reith CA, Sillar KT (1999) Development and role of $\mathrm{GABA}_{\mathrm{A}}$ receptormediated synaptic potentials during swimming in postembryonic Xenopus laevis tadpoles. J Neurophysiol 82:3175-3187.

Roberts A, Clarke JDW (1982) The neuroanatomy of an amphibian embryo spinal cord. Philos Trans R Soc Lond B Biol Sci 296:195-212.

Roberts A, Dale N, Ottersen OP, Storm-Mathisen J (1987) The early development of neurons with GABA immunoreactivity in the CNS of Xenopus laevis embryos. J Comp Neurol 261:435-449.

Sasaki M, Gonzalez-Zulueta M, Huang H, Herring WJ, Ahn S, Ginty DD, Dawson VL, Dawson TM (2000) Dynamic regulation of neuronal NO synthase transcription by calcium influx through a CREB family transcription factor-dependent mechanism. Proc Natl Acad Sci USA 97:8617-8622.

Scrymgeour-Wedderburn JF, Reith CA, Sillar KT (1997) Voltage oscillations in Xenopus spinal cord neurons: developmental onset and dependence on co-activation of NMDA and 5-HT receptors. Eur J Neurosci 9:1473-1482.

Sillar KT, Simmers AJ, Wedderburn JFS (1992a) The post-embryonic development of cell properties and synaptic drive underlying locomotor rhythm generation in Xenopus larvae. Proc R Soc Lond B Biol Sci 249:65-70.

Sillar KT, Wedderburn JFS, Simmers AJ (1992b) Modulation of swimming rhythmicity by 5-hydroxytryptamine during postembryonic development in Xenopus laevis. Proc R Soc Lond B Biol Sci 250:107-114.

Sillar KT, Woolston A-M, Wedderburn JFS (1995) The involvement of brainstem serotonergic interneurons in the development of a vertebrate spinal locomotor circuit. Proc R Soc Lond B Biol Sci 259:65-70.

Soffe SR (1987) Ionic and pharmacological properties of reciprocal inhibition in Xenopus embryo motoneurones. J Physiol (Lond) 382:463473.

Sun Q-Q, Dale N (1998) Developmental changes in expression of ion currents accompany maturation of locomotor pattern in frog tadpoles. J Physiol (Lond) 507:257-264.

Talley EM, Lei QB, Sirois JE, Bayliss DA (2000) TASK-1, a two-poredomain $\mathrm{K}^{+}$channel, is modulated by multiple neurotransmitters in motoneurons. Neuron 25:399-410.

Talley EM, Solórzano G, Lei Q, Kim D, Bayliss DA (2001) CNS distribution of members of the two-pore-domain (KCNK) potassium channel family. J Neurosci 21:7491-7505.

van Mier P, Joosten HWJ, van Reden R, ten Donkelaar HJ (1986) The development of serotonergic raphespinal projections in Xenopus laevis. Int J Dev Neurosci 4:465-476.

Vinay L, Brocard F, Pflieger J-F, Simeoni-Alias J, Clarac F (2000) Prenatal development of lumbar motoneurons and their inputs in the rat. Brain Res Bull 53:635-647.

Vizi ES (1984) Non-synaptic interactions between neurons: modulation of neurochemical transmission. Chichester, UK: Wiley.

Wall MJ, Dale N (1993) $\mathrm{GABA}_{\mathrm{A}}$ receptors modulate glycinergic inhibition and spike threshold in Xenopus embryo spinal neurones. J Physiol (Lond) 469:275-290.

Wall MJ, Dale N (1995) A slowly activating $\mathrm{Ca}^{2+}$-dependent $\mathrm{K}^{+}$current that plays a role in termination of swimming in Xenopus embryos. J Physiol (Lond) 487:557-572. 